\title{
Evaluation of rosacea before and after intense pulsed laser treatment using reflectance confocal microscopy: case report
}

\begin{abstract}
Erythematotelangiectatic rosacea is a common, benign, inflammatory skin condition that is typified by centrofacial erythema and flushing with telangiectatic vessels on the cheeks that intensifies with particular stimuli. Characteristics of rosacea on reflectance confocal microscopy have been described in the literature, but RCM has not yet been used to evaluate findings both before and after the cosmetic treatment modality intense pulsed laser. We describe our findings in a case of facial rosacea before treatment and assess post-IPL treatment success with reflectance confocal microscopy.
\end{abstract}

Keywords: rosacea, intense pulsed laser, reflectance confocal microscopy, demodex
Volume 2 Issue 4 - 2018

\section{Gina Francisco,Attiya Haroon, Jisun Cha, Babar Rao}

Department of Dermatology, Rutgers Robert Wood Johnson Medical School, USA

\section{Correspondence: Gina Francisco MBS, Department of} Dermatology, Rutgers Robert Wood Johnson Medical School, I World's Fair Drive, Somerset, NJ, 08873, USA, Tel 73289560 09, Email gfranci9@rwjms.rutgers.edu

\begin{abstract}
Abbreviations: IPL, intense pulsed laser; RCM, reflectance confocal microscopy

\section{Introduction}

Reflectance confocal microscopy is a real-time non-invasive diagnostic imaging technique used in the field of dermatology to diagnose and monitor neoplastic, inflammatory, and infectious lesions. This in vivo technique images the skin up to a depth of 200-300 $\mu \mathrm{m}$ and obtains horizontal en face images of the epidermis and upper dermis with a resolution comparable to light microscopy. In vivo examinations before and after laser treatment of benign, yet cosmetically-concerning lesions, such as straie distensae, acne scars and melasma, have been described in the literature. ${ }^{1-5}$ Rosacea, a similar dermatologic complaint, has a prevalence of $0.09-2.7 \%$ in the general population. ${ }^{6}$ There are four recognized subtypes: erythematotelangiectatic, papulopustular, phymatous and ocular, that may overlap. Rosacea negatively impacts quality of life and patients with the condition are more likely to experience depression, embarrassment, social phobia, and stress than patients without the disease. ${ }^{7}$ Attempts at treatment often fail, as the true etiology and pathophysiology remain unidentified.
\end{abstract}

It is theorized that an abnormal proliferation of Demodex folliculorum mites within the skin plays a pathogenic role in rosacea ${ }^{8,9}$ Rosacea has been evaluated by RCM in previous studies through non-invasive identification and quantification of Demodex mites from the surface of the skin to the upper part of hair follicles, about 100-120micrometers deep. Treatment response was categorized as alleviation of symptoms and reduction in number or total clearance of mites, typically after topical and systemic acaricidal therapy. ${ }^{10,11}$ Intense pulsed laser is an additional treatment modality for the persistent erythema, telangiectasia, and flushing associated with rosacea. IPL has demonstrated cosmetic efficacy and is associated with a decreased rate of post-procedural purpura when compared to other vascular lasers. ${ }^{12,13}$ Drawbacks to using IPL include that 3-7 treatments about 1 month apart are typically needed to see clinical improvement. In addition, patients pay for this cosmetic procedure out-of-pocket and frustration may occur when anticipated results are not evident instantaneously.
Although scales such as the Spectrophotometric Erythema Score help to categorize and gauge treatment response, ${ }^{13} \mathrm{RCM}$ can be used as an objective measure of the effects of IPL treatment on human skin. ${ }^{14}$ Specific features of rosacea on RCM include enlarged tortuous capillaries, a perifollicular and perivascular inflammatory infiltrate, and an increased diameter of the pilosebaceous ducts. ${ }^{15,16}$ On RCM, capillary loops are seen in the center of dermal papillae as black holes, often showing highly-reflective erythrocytes rolling within them. ${ }^{15}$ We describe the findings in a case of erythematotelangiectatic rosacea before and after two rounds of intense pulsed laser treatment.

\section{Report of a case}

A 50-year-old female presented to our clinic with centrofacial and perioral erythema with telangiectatic vessels on the cheeks that would periodically intensify with exercise and heat (Figure 1A). Five years prior to presentation, she underwent 5rounds of intense pulsed laser treatment at an outside dermatologist with temporary (2 year) resolution of symptoms, so it was decided to initiate IPL therapy treatment with a starting fluence of $23 \mathrm{~J} / \mathrm{cm}^{2}$. Four weeks after her second round of IPL, the patient continued to notice facial redness and experience flushing. Reflectance confocal microscopy using the Vivascope 1500 was performed on the cheek in order to confirm the diagnosis and assess characteristics. A small drop of oil was applied to the target area of the face and a metal ring with polymer window was attached to the skin. The metal ring was then docked magnetically with the RCM scope head. RCM images composed of 3 cubes and 1 stack were obtained at 20 and 40micrometers and were stitched together to form a mosaic image.

An RCM image taken of the cheek prior to any treatment (Figure 1B) shows an increased amount of tortuous capillary loops, an increased diameter of the pilosebaceous ducts, and a marked inflammatory infiltrate. These findings are consistent with rosacea. ${ }^{15,16}$ After two rounds of IPL, erythema of the cheeks was still present four weeks later with a fewer number of telangiectatic vessels, as seen on dermoscopy (Figure 2A). RCM imaging was suggestive of treatment response, depicting a decreased number of capillary loops and a decreased diameter of the pilosebaceous ducts. Inflammatory cells are still present, although sparse (Figure 2B). 


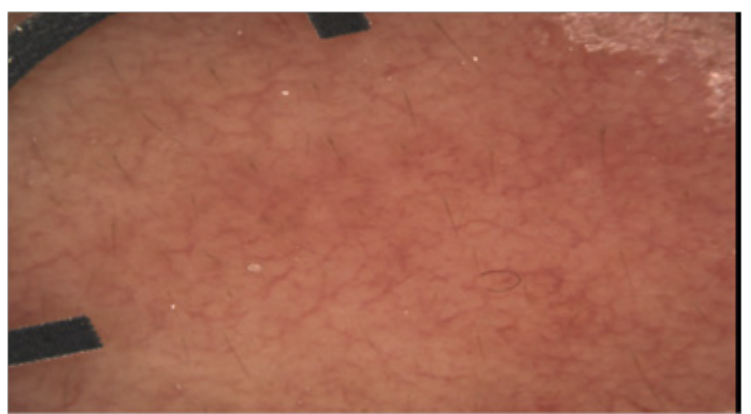

I(A)

Figure IA Before treatment. Dermoscopic image of background erythema and telangiectatic vessels on the cheek.

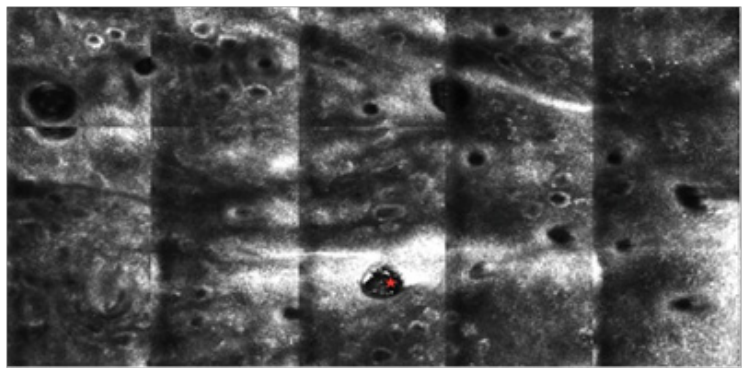

I(B)

Figure IB Before treatment. Reflectance confocal microscopic image of erythematotelangiectatic rosacea showing increased vascularity dilated follicular ostia with hyper-reflective Demodex mites (red star) and small bright cells representing inflammation.

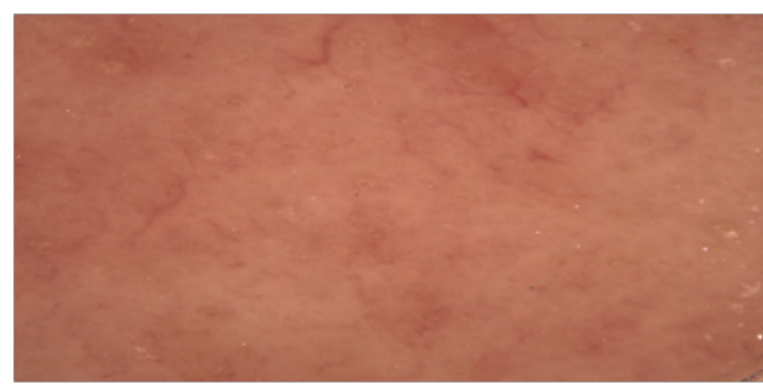

2(A)

Figure 2(A) After treatment. Dermoscopic image showing decreased erythema and number of telangiectatic vessels.

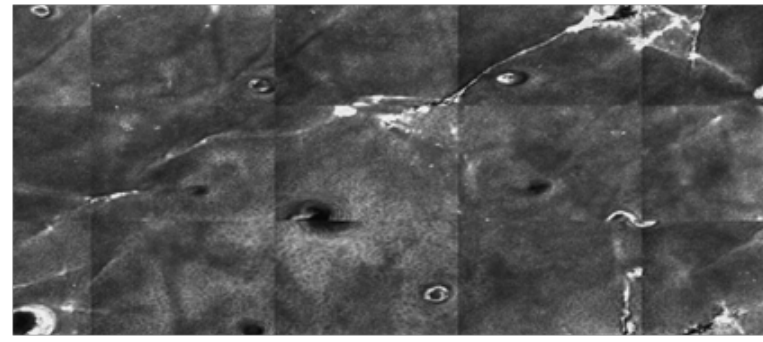

2(B)

Figure 2B Reflectance confocal microscopic image showing a decrease in the number of dilated capillary loops and decreased width of pilosebaceous ducts. Minimal inflammatory cells are present. There is no sign of demodex mite infestation within the hair follicles.

\section{Conclusion}

Reflectance confocal microscopy can be used as an adjunct in the diagnosis and assessment of treatment response of erythematotelangiectatic rosacea. It may be difficult to make a definitive diagnosis of rosacea in cases that manifest clinically as a similar inflammatory condition, such as seborrheic dermatitis. Pretreatment RCM findings in this patient included enlarged tortuous capillaries, an inflammatory infiltrate, presence of demodex mites, and increased diameter of the pilosebaceous ducts, which aided in the confirmation of the diagnosis. After treatment, there was a decrease in the number of dilated capillary loops and a decreased width of the pilosebaceous ducts. In addition, there was no sign of demodex mite infestation within the hair follicular unit. RCM can be useful in the assessment of treatment response, and it may allow the physician to recommend continuation of IPL treatment based off of objective findings in cases where the clinical response is gradual.

\section{Acknowledgements}

No authors received financial or other compensation for their contributions.

\section{Conflict of interest}

Authors declares that there is no conflict of interest.

\section{References}

1. Guida S, Galimberti MG, Bencini M, et al. Treatment of striae distensae with non-ablative fractional laser: clinical and in vivo microscopic documentation of treatment efficacy. Lasers Med Sci. 2018;33(1):75-78.

2. Bertin C, Lopes-DaCunha A, Nkengne A, et al. Striae distensae are characterized by distinct microstructural features as measured by noninvasive methods in vivo. Skin Res Technol. 2014;20(1):81-86.

3. Bencini PL, Tourlaki A, Galimberti M, et al. Nonablative fractional photothermolysis for acne scars: clinical and in vivo microscopic documentation of treatment efficacy. Dermatol Ther. 2012;25(5):463-467.

4. Ardigo M, Cameli N, Berardesca E, et al. Characterization and evaluation of pigment distribution and response to therapy in melasma using in vivo reflectance confocal microscopy: a preliminary study. J Eur Acad Dermatol Venereol. 2010;24(11):1296-1303.

5. Kang HY, Bahadoran P, Suzuki I, et al. In vivo reflectance confocal microscopy detects pigmentary changes in melasma at a cellular level resolution. Exp Dermatol. 2010;19(8): e228-e233.

6. Tan J, Berg M. Rosacea: current state of epidemiology. J Am Acad Dermatol. 2013;69(6 Suppl 1):S27-S35.

7. Moustafa F, Lewallen RS, Feldman SR. The psychological impact of rosacea and the influence of current management options. J Am Acad Dermatol. 2014;71(5):973-980.

8. Bonnar E Eustace P, Powell FC. The Demodex mite population in rosacea. J Am Acad Dermatol. 1993;28(3):443-448.

9. Harmelin Y, Delaunay P, Erfan N, et al. Interest of confocal laser scanning microscopy for the diagnosis and treatment monitoring of demodicosis. $J$ Eur Acad Dermatol Venereol. 2014;28(2):255-257.

10. Turgut Erdemir A, Gurel MS, Koku Aksu AE, et al. Reflectance confocal microscopy vs. standardized skin surface biopsy for measuring the density of Demodex mites. Skin Res Technol. 2014;20(4):435-439.

11. Sattler EC, Hoffmann VS, Ruzicka T, et al. Reflectance confocal microscopy for monitoring the density of Demodex mites in patients with rosacea before and after treatment. Br J Dermatol. 2015;173(1):69-75. 
12. Papageorgiou P, Clayton W, Norwood S, et al. Treatment of rosacea with intense pulsed light: significant improvement and long-lasting results. $\mathrm{Br}$ J Dermatol. 2008;159(3):628-632.

13. Neuhaus IM, Zane LT, Tope WD. Comparative efficacy of nonpurpuragenic pulsed dye laser and intense pulsed light for erythematotelangiectatic rosacea. Dermatol Surg. 2009;35(6):920-928.

14. Sun W, Wu J, Qian H, et al. Objective evaluation of the effects of intense pulsed light treatment on Asian skin by reflectance confocal microscopy analysis. Lasers Med Sci. 2018;33(4):779-784.
15. Gonzalez S, Gilaberte-Calzada Y. In vivo reflectance-mode confocal microscopy in clinical dermatology and cosmetology. Int J Cosmet Sci. 2008;30(1):1-17.

16. Białek-Galas K, Wielowieyska-Szybińska D, Dyduch G, et al. The use of reflectance confocal microscopy in selected inflammatory skin diseases. Pol J Pathol. 2015;66(2):103-108. 